\title{
Report of ICCM 2013 at Taipei
}

\author{
by Jing Yu
}

\author{
Department of Mathematics, National Taiwan University
}

The sixth International Congress of Chinese Mathematicians (ICCM) was held from July 14 to July 19, 2013, in Taipei Taiwan. The opening ceremony took place at the Grand Hotel of Taipei on July 14 Sunday, with about one thousand participants from all over the world. This monumental activity of the whole Chinese mathematical community started with welcome greetings by the ICCM honorary chair Professor Shing-Tung Yau from Harvard University, President Pan-Chyr Yang of National Taiwan University, Mr. Ronnie C. Chan, co-founder of the Morningside Foundation and Chairman of Hang Lung Properties, and Professor Jing Yu from the Taida Institute of Mathematical Sciences who also served as chairman of the local organizing committee. This was the second time that ICCM held in Taiwan, first time was 12 years ago. Despite an unexpected Typhoon invasion to the Taiwan Island on July $11 / 12$, we are very happy to see that almost all the participants this year who scheduled to join managed to do so eventually.

As in each ICCM, people are able to witness the exciting progresses of mathematics made by Chinese mathematicians just recently.

Following now the tradition of ICCM, the big event in the opening ceremony was the Awards presentation, in particular the Morningside Medals. This year, beside the Morningside Gold and Silver Medal, a special Morningside Achievement Award was awarded to Yitang Zhang from the New Hampshire University, for his monumental breakthrough in Number Theory, proving the abundance of bounded gaps between consecutive primes. This brilliant success throws new lights on the entire development of analytic number theory. Another medal first awarded this year is the Morningside Mentor Award in Mathematics, given to Chengbiao Pan, a retired professor from China Agricultural University who held adjoint position at Peking university for many years.

There are two 2013 Morningside Gold medals. One was awarded to Xuhua He of HKUST for his fundamental contributions to arithmetic geometry, algebraic groups, and representation theory. The other Gold Medal awarded to Ye Tian, AMSS of the Chinese Academy of Sciences, for his deep and original work on the congruence number problem. The 2013 Morningside medal of applied mathematics was awarded to Xianfeng David Gu, for his contributions to computational geometry, imaging, and graphics.
There are four Morningside Silver Medals awarded this year. The recipients are Chieh-Yu Chang of National Tsing Hua University, Xiaoqing Li of State University of New York at Buffalo, Hao Xu of Harvard University, and Tai-Peng Tsai of the University of British Columbia.

Professor Jean-Pierre Serre at the College de France, Fields Medalist, also recipient of both the Abel Prize, and the Wolf prize, was awarded the 2013 ICCM International Cooperation Award. Professor Serre has been visiting

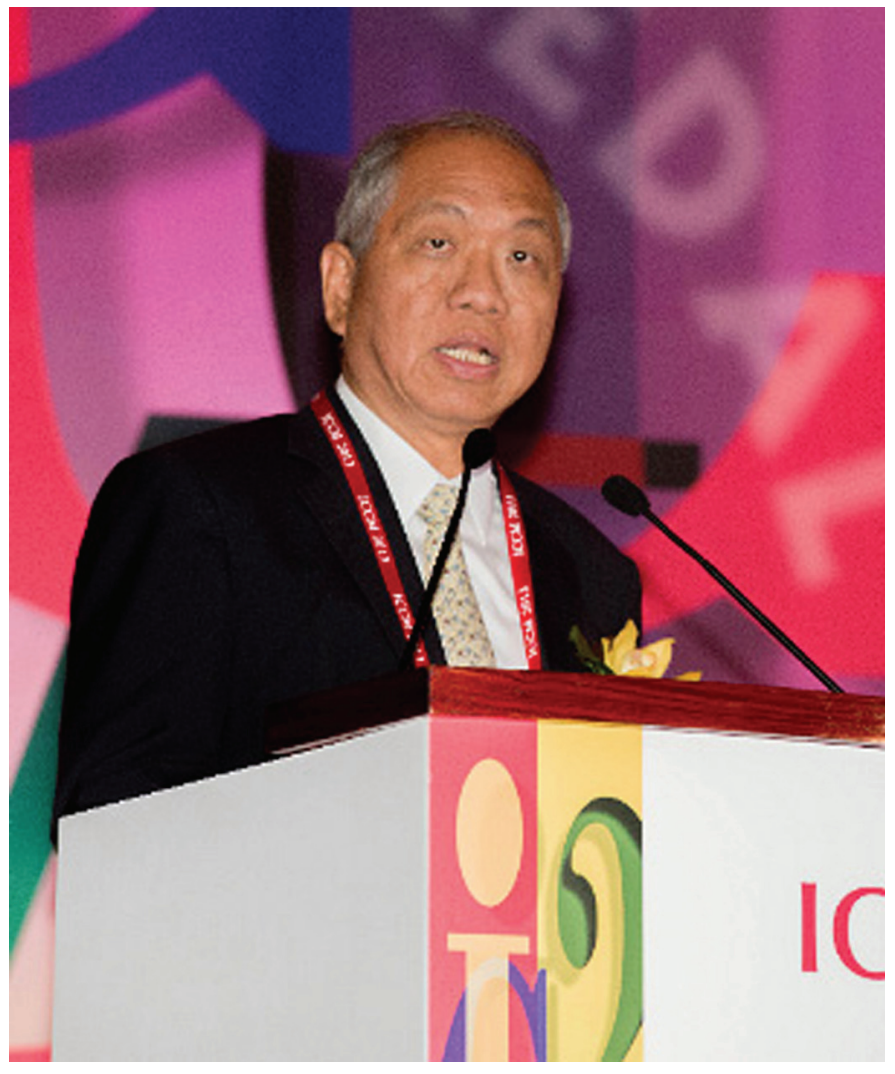

Shing-Tung Yau

Taiwan biannually since 2009, and greatly impacted and encouraged many young mathematicians.

The two 2013 Chern Prize recipients are former President Si-Chen Li of the National Taiwan University, for public service in support of mathematics, and Professor Bong Lian of Brandeis University, for fundamental contributions to mathematical physics. 
The July 14 afternoon activity at the Grand hotel started with a Symposium, Unlocking our future: how government policies can foster fundamental science development. This symposium has Dr. Gerald L. Chan, co-founder of Morningside, as moderator, with panelists: Chi-Huey Wong, the President of the Academia Sinica, Yan-Hwa Wu, President of National Chiao Tung University, and Way Kuo, President, President of City University of Hong Kong. The second part of this Grand Hotel Symposium consist of two fascinating talks aiming at general audience, "the art of bridge building" delivered by Professor C.L. Liu of the National Tsing Hua University, and

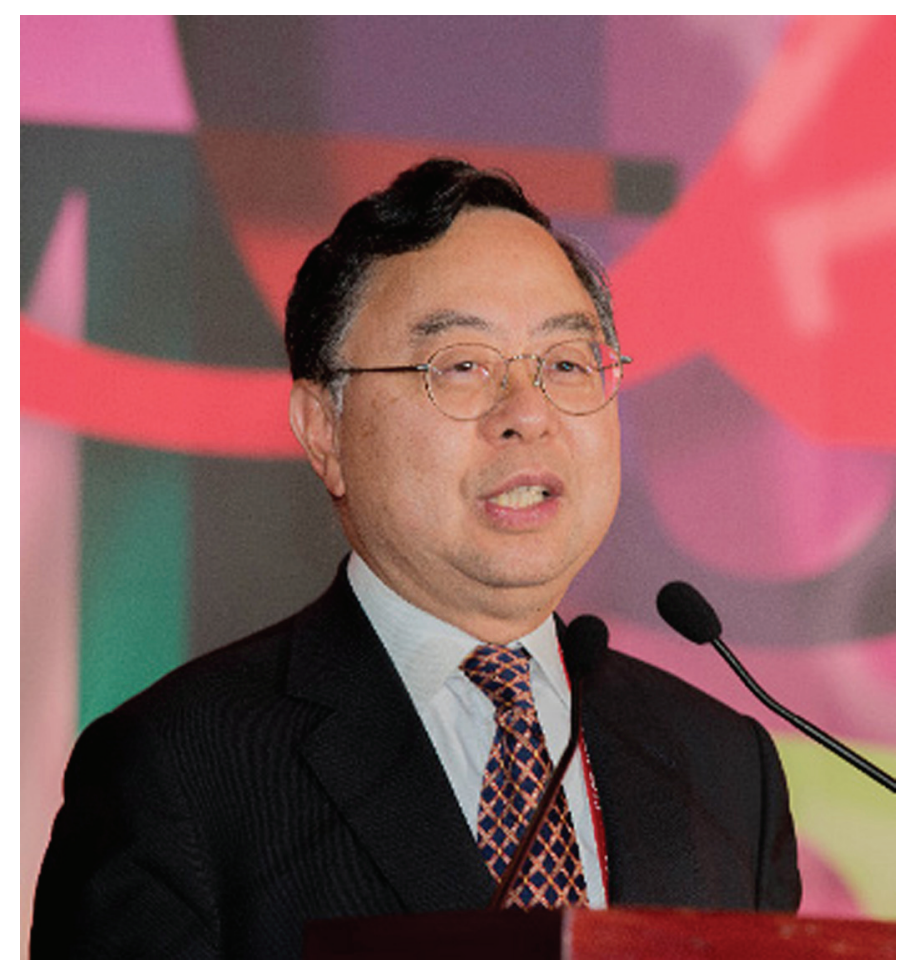

Ronnie C. Chan

"juggling mathematics and magic" delivered by Professor Ronald Graham of UC San Diego.

Honorary guest Jin-Pyng Wang, President of the Legislative Yuan of Taiwan, joined the ICCM opening activity at the Grand Hotel in the evening of the opening day. Besides greeting the morning ICCM awardee, he also took part in the 2013 New World Mathematics Awards presentation ceremony. The New World Awards presentation is another tradition of ICCM, sponsored by the New World Development Company Limited, awarding prizes to the best recent Ph.D. Thesis, Master Thesis, and Bachelor Thesis in mathematics. July 14 evening, this award was presented to 27 students, among them are 7 gold Prizes for Doctor Thesis.

Venue of 2013 ICCM after the first day is sited on the Campus of NTU National Taiwan University, in a set of comfortable well-equipped lecture rooms that is situated in the beautiful drunken moon lakeside. Every morning of July 15-19, ICCM began with a distinguished one hour lecture by an international honorary guest: Jean-Pierre Serre on historical remarks of cohomology, John H. Coates on the Conjecture of Birch and Sinnerton-Dyer, Dorian Goldfeld on the relative trace formula for unipotent groups, Björn Engquist on multiscale simulations of dynamical systems with oscillatory solutions, and Stanley Osher on what sparsity and 11 optimization can do for you. These talks are followed by the one hour plenary lectures, there are six such lectures every day, covering a broad landscape of both pure and applied mathematics, and addressing to the most important recent progresses. The abstracts of all these inspiring lectures are appended with this report. We are editing a more complete account of these lectures into Proceedings of the Six ICCM to be published in near future.

There were 152 invited 45-minute talks, dividing into 10 sections:

1. Number Theory, Automorphic Forms, and Arithmetic Geometry, 25 talks.

2. Algebra, History of Mathematics, 9 talks.

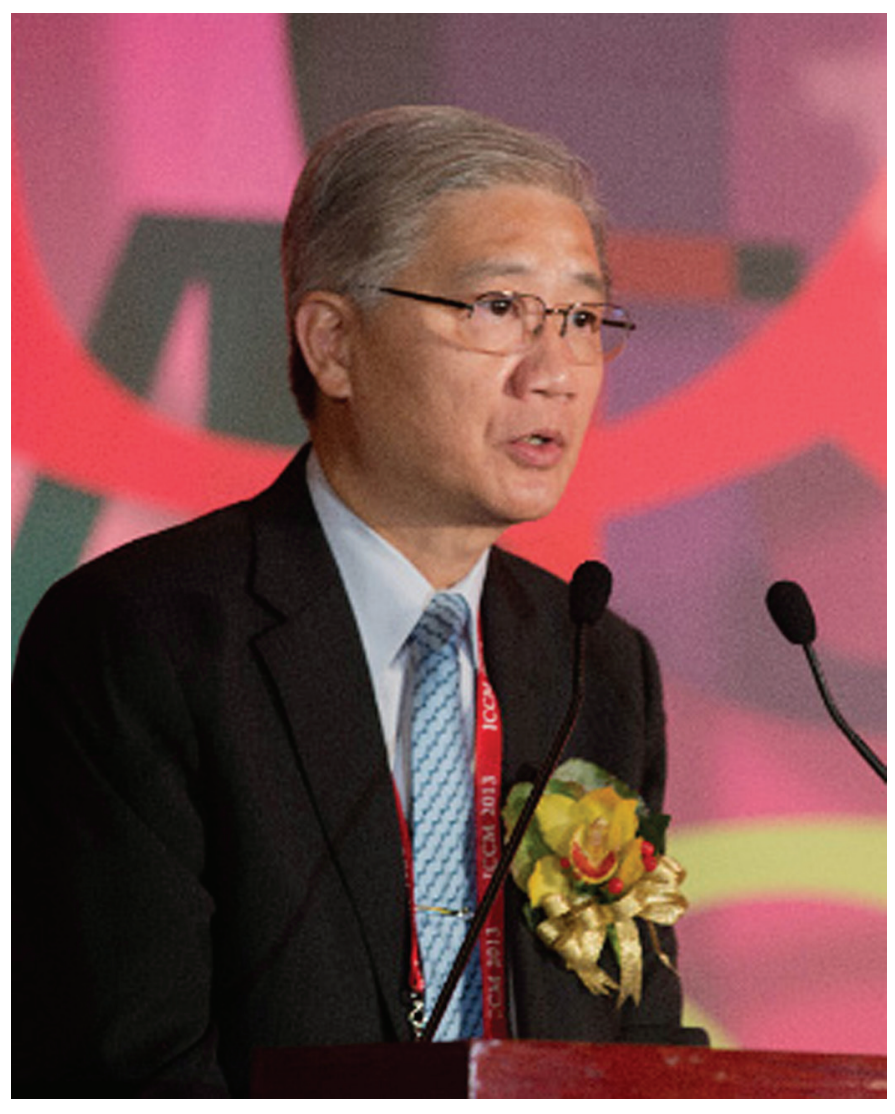

Pan-Chyr Yang

3. Discrete Mathematics, Combinatorics, 8 talks.

4. Algebraic Geometry, Complex Geometry, 18 talks.

5. Geometric Analysis, Differential Geometry, Symplectic Geometry, 17 talks. 
6. Topology, Geometry and Mathematical Physics, 17 talks.

7. Statistical Theory, Methods, Applications, Probability, Financial Mathematics, 11 talks.

8. Dynamical Systems, Control Theory, Optimization, Functional Analysis, Fractals, 18 talks.

9. Computational Mathematics, Imaging, Mathematical Biology, 17 talks.

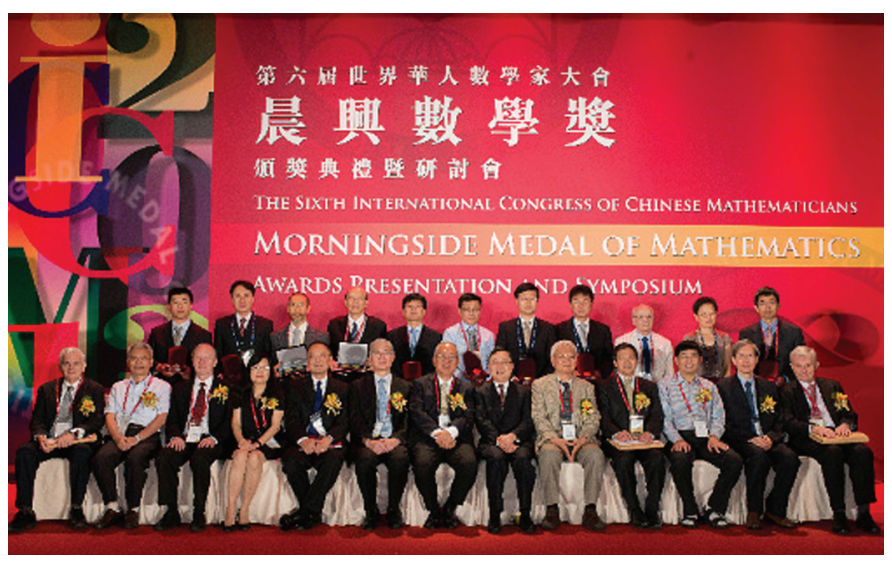

Morningside Medalists

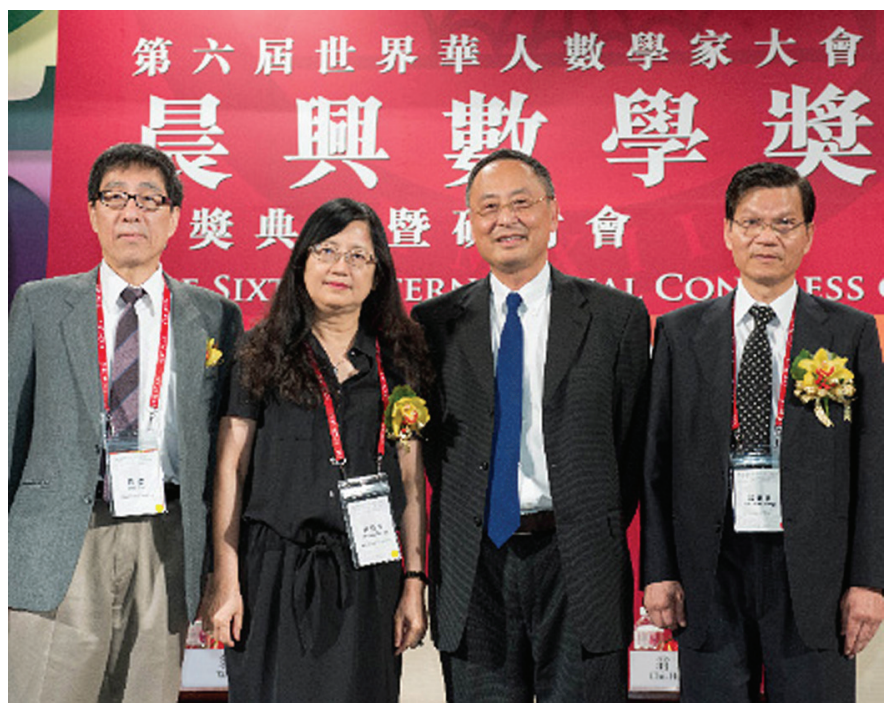

Way Kuo, Yan-Hwa Wu, Gerald L. Chan, and Chi-Huey Wong

10. Partial Differential Equations, 12 talks.

There were also 96 contributed talks, each given 20 minutes.

Among the participants of the six ICCM, there are over 400 coming from mainland China, it took lots efforts to process their travel documents to Taiwan. Although there are now direct flights from all major cities of mainland to Taiwan, the travel documents still have to be approved by authorities from both China and Taiwan which is certainly time consuming. ICCM is by far the largest scale academic interaction across the strait. We are able to manage it this time without genuine obstacles.

The sponsoring organizations of the 2013 ICCM Conference include: National Taiwan University (Taida Institute for Mathematical Sciences, Center of Advanced Studies for Theoretical Sciences, Department of Mathematics), National Science Council (National Center for Theoretical Sciences, Department of International Cooperation), Ministry of Economic Affairs, the Yau Center of National Chiao Tung University, and the Taipei City Government.

\section{Abstracts of the Special Lecture, Morningside Lectures, and Plenary Lectures}

\section{Special Lecture}

\section{Historical remarks on cohomology \\ Jean-Pierre Serre \\ Collège de France}

Abstract. The introduction of cohomological methods in algebra, complex variables, algebraic geometry and number theory is one of the characteristics of last century mathematics. The lecture will describe how some of these methods came into being, with special emphasis on étale cohomology and Weil conjectures.

\section{Morningside Lectures}

\section{On the conjecture of Birch and Swinnerton-Dyer}

John H. Coates

University of Cambridge

Abstract. The lecture will discuss the important progress on the conjecture of Birch and Swinnerton-Dyer made by Tian in his recent work on the classical congruent number problem, and its possible generalization to other elliptic curves.

\section{The relative trace formula for unipotent subgroups}

Dorian Goldfeld Columbia University

Abstract. The relative trace formula of Jacquet has played a fundamental role in recent advances in number theory. The goal of my lecture will be to explain and introduce a very explicit version of the relative trace formula for the quotient $U \backslash G / U$ when $G=G L(n)$ and $U$ is a unipotent subgroup. In the second part of the lecture, I will show how to obtain applications of the relative trace formula to some well known problems in analytic number theory. 


\section{Multiscale simulations of dynamical systems with oscillatory solutions}

Björn Engquist

The University of Texas at Austin

Abstract. The heterogeneous multiscale method is a framework that can be used to design and analyze numerical methods for stiff ordinary differential equations with oscillatory solutions. Microscale simulations are applied in subsets of the computational domain in order to approximate the averaged dynamical system. We will discuss the difficulty of finding macroscale variables and present new techniques for avoiding this difficulty. We

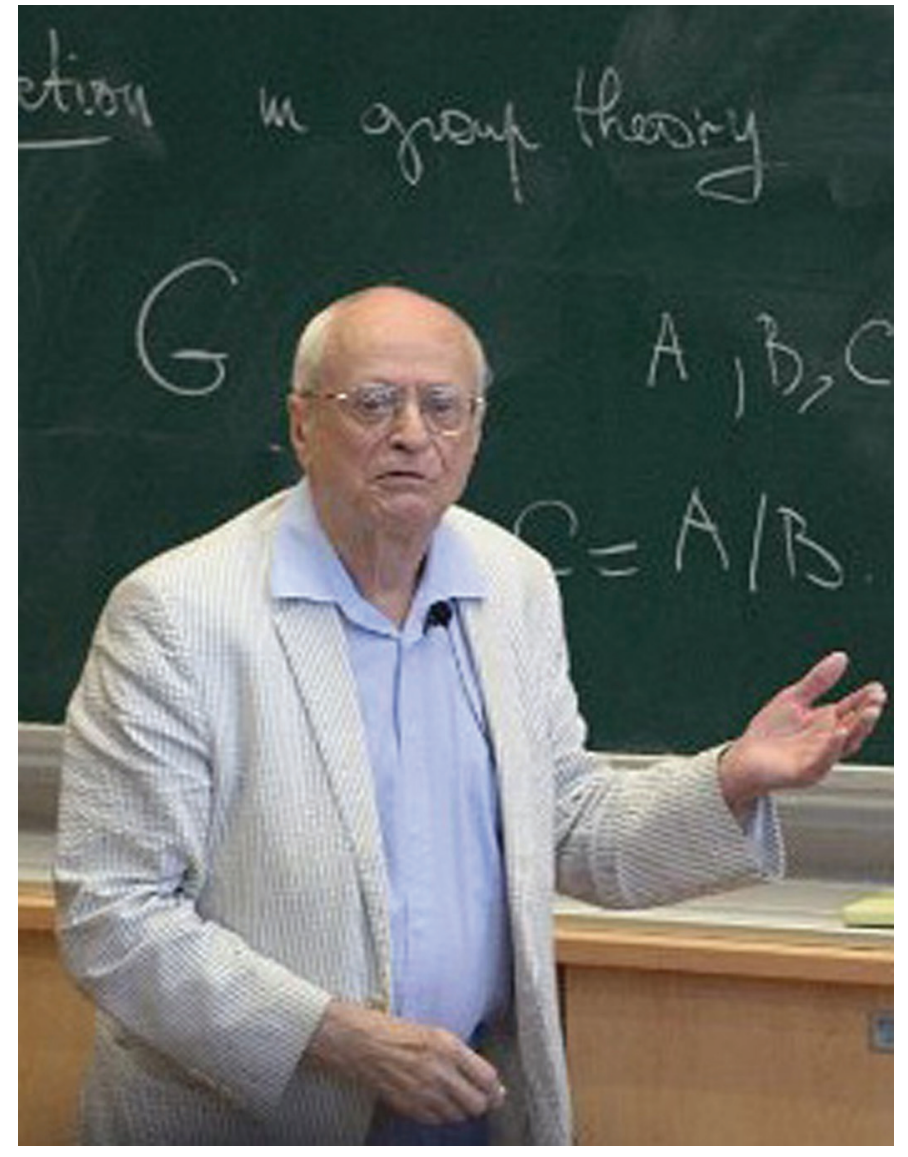

Jean-Pierre Serre

will compare with related methods and give numerical examples.

\section{What sparsity and 11 optimization can do for you}

Stanley Osher

University of California, Los Angeles

Abstract. Sparsity and compressive sensing have had a tremendous impact in science, technology, medicine, imaging, machine learning and now, in solving multiscale problems in applied partial differential equations, developing sparse bases for Elliptic eigenspaces and connections with viscosity solutions to Hamilton-Jacobi equations.

11 and related optimization solvers are a key tool in this area. The special nature of this functional allows for very fast solvers: $\mathrm{ll}$ actually forgives and forgets errors in Bregman iterative methods.

I will describe simple, fast algorithms and new applications ranging from sparse dynamics for PDE, new regularization paths for logistic regression and support vector machine to optimal data collection and hyperspectral image processing. This is joint work with many people.

\section{Plenary Lectures}

\section{Leaves and local Hecke symmetry}

Ching-Li Chai

Institute of Mathematics, Academia Sinica/University of Pennsylvania

Abstract. Let $M$ be a PEL type moduli space over an algebraic closure of the prime field with p elements; points of such a moduli space corresponds to isomorphism classes of abelian varieties with prescribed symmetries of a fixed type. A leaf in $M$ is the locally closed subvariety obtained by fixing all $p$-adic invariants of these PEL type abelian varieties. A conjecture of Oort predicts that every prime-to-p Hecke orbit in $\mathrm{M}$ is Zariski dense in the leaf containing this Hecke orbit. In this talk we will present an overview of questions and results related to this conjecture, as well as recent progress in two aspects.

The first is a scheme-theoretic definition of the notion of leaves, more than a dozen years after its birth.

The new definition allows a natural construction of group-like structures on the formal completion at a point of a leaf, generalizing the Serre-Tate canonical coordinates for ordinary abelian varieties.

The second is a method for computing a weak asymptotic expansion of the action of the local stabilizer subgroup on the formal completion of a point of $M$.

This method will be illustrated in the case of the Lubin-Tate action, which occurs in moduli spaces of PEL type $U(n, 1)$.

\section{Parameter estimation in systems and control}

Han-Fu Chen

Academy of Mathematics and Systems Science, Chinese Academy of Sciences

Abstract. Many problems from systems and control such as system identification for linear systems and for a large class of nonlinear systems as well, adaptive regulation and iterative learning control (ILC) for stochastic systems, principal component analysis, adaptive filtering, blind identification in communication, and a certain kind of problems from networked systems and signal processing and many others can be reduced to problems of 
parameter estimation. The unknown parameters may be viewed as roots of functions, and parameter estimation can be transformed to a root-seeking problem based on observations on an unknown function called as the regression function. However, the resulting root-seeking problem can hardly be solved by the classical Robbins-Monro (RM) algorithm, because for its convergence a set of restrictive conditions are required.

The stochastic approximation (SA) algorithm with expanding truncations (SAAWET) is then introduced, and for its convergence a general convergence theorem (GCT) is presented. SAAWET is successfully applied to solving problems from systems and control. As a matter of fact,

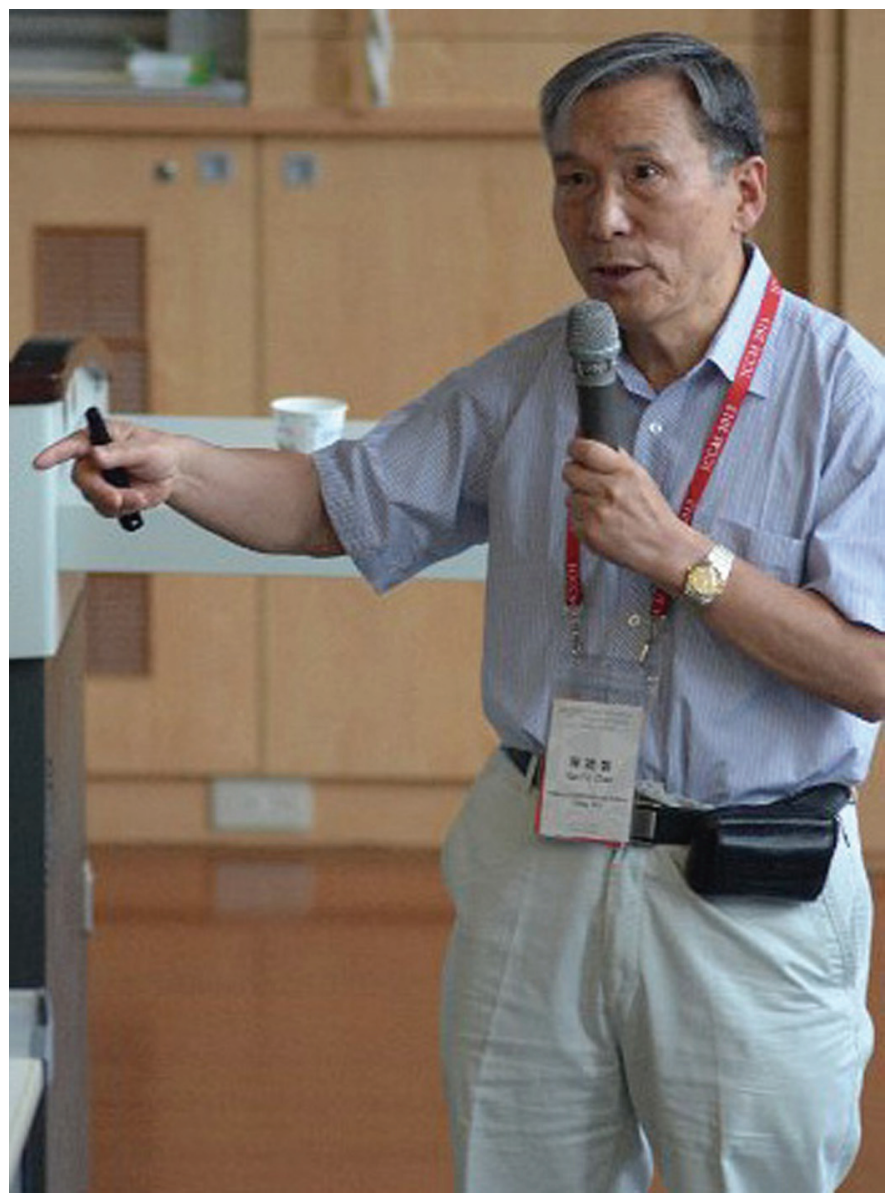

Han-Fu Chen

all problems listed above have been solved under reasonable and checkable conditions. Moreover, all solutions are provided in a recursive way, which is convenient for applications. This approach suggests solving the problems by two steps: first, transform problems to estimation of parameters or to root-seeking problems with appropriately selected regression function, then apply SAAWET with emphasis on proving satisfaction of the conditions required by GCT.

To demonstrate such an approach to solving problems from systems and control, the identification of the multivariate Hammerstein system is analyzed, and the recursive identification algorithms for both the linear and the nonlinear parts of the system are presented. All estimates given by the recursive algorithms are strongly consistent under reasonable conditions. Finally, the proposed algorithms are used to numerically identify a two-dimensional Hammerstein system. It is shown that the simulation results are consistent with the theoretical analysis.

\section{Sixty years of compactifications of $\mathbb{C}^{n}$ \\ Baohua $\mathrm{Fu}$ \\ Academy of Mathematics and Systems Science, Chinese Academy of Sciences}

Abstract. I shall give an overview of the study of compactifications of $\mathbb{C}^{n}$ as projective algebraic manifolds with $b_{2}=1$, a problem asked by Hirzebruch in 1954. Recent progress on equivariant compactifications will also be discussed. This is based on joint works with Jun-Muk Hwang.

\section{Sliced inverse regression with interaction detection \\ Jun Liu \\ Harvard University}

Abstract. Previously we have proposed a Bayesian partition model for detecting interactive variables in a classification setting with discrete covariates. This framework takes advantage of the structure of the naive Bayes classifier and introduces latent indicator variables for selecting variables and interactions. In our effort to extend the methods to continuous covariates, we found interesting connections with semi-parametric index models and the Sliced Inverse Regression method. In index models, the response is influenced by the covariates through an unknown function of several linear combinations of the predictors. Our finding of the Bayesian formulation of such models enabled us to propose a set of new models and methods that can effectively discover second-order effects and interactions among the covariates. A two-stage stepwise procedure based on likelihood ratio test is developed to select relevant predictors and a Bayesian model with dynamic slicing scheme is derived. The performance of the proposed procedure in comparison with some existing method is demonstrated through simulation studies. This is based on the joint work with Bo Jiang.

\section{Automorphic Forms and the Langlands program for covering groups}

Wee Teck Gan

National University of Singapore

Abstract. In this talk, I will survey some recent results (by many people) about a possible extension of the Langlands program to a class of covering groups studied by Beylinski and Deligne. These include the definition of the L-group of a covering group, the classification of local 
representations, the theory of the trace formula and endoscopy and the study of the automorphic discrete spectrum.

\section{Ricci flow and classification of certain 4-manifolds}

Bing-Long Chen

Sun Yat-sen University

Abstract. It has been well-known that Ricci flow has played a vital role in 3-dimensional topology. The Ricci flow with surgery in dimension 4 has already been developed in a seminal paper by R. Hamilton in 1997. It was expected that Ricci flow may also exert a great role in 4-dimensional geometry and topology. I hope this talk will serve as a step toward this subject. The main result of this talk, obtained from the study of the Ricci flow, is to show a complete classification of compact 4-manifolds whose certain generalized Yamabe invariants are positive. This classification is an analogue of a classification theorem of R. Schoen and S.T. Yau in 1970's on 3-manifolds with positive ordinary Yamabe invariants. This lecture is based on author's two joint papers with X.P. Zhu, one joint paper with S.H. Tang and X.P. Zhu.

\section{Mathematics of shock reflection-diffraction and von Neumann's conjectures}

Gui-Qiang G. Chen

University of Oxford

Abstract. Shock waves are fundamental in nature. When a shock impinges an obstacle (steady or flying), shock reflection-diffraction phenomena occur. One of the most fundamental problems in mathematical fluid mechanics is shock reflection-diffraction by wedges. The complexity of reflection-diffraction configurations was first reported by Ernst Mach in 1878, who first observed two patterns of configurations. The problems remained dormant until the 1940s when John von Neumann, as well as other mathematical/experimental scientists, began extensive research into all aspects of shock reflection-diffraction phenomena. Moreover, the reflection-diffraction configurations are the core configurations in the structure of multidimensional Riemann solutions, which are building blocks and local structure of general entropy solutions and determine global attractors and asymptotic states of the solutions for multidimensional hyperbolic systems of conservation laws.

The shock reflection-diffraction problems involve several core mathematical difficulties we have to face in solving nonlinear partial differential equations in mechanics and geometry. These include nonlinear equations of mixed hyperbolic-elliptic type, nonlinear degenerate elliptic equations, nonlinear degenerate hyperbolic equations, free boundary problems for nonlinear degenerate equations, corner singularity/regularity especially when free boundaries meet degenerate curves, a priori estimate techniques, among others.
In this talk we will start with various shock reflection-diffraction phenomena and historic perspectives, their fundamental scientific issues, and their theoretical roles in the mathematical theory of multidimensional hyperbolic systems of conservation laws. Then we will describe how the global shock reflection-diffraction problems can be formulate as free boundary problems for nonlinear conservation laws of mixed hyperbolic-elliptic type. Finally we will discuss some recent developments in solving von Neumann's conjectures and establishing a mathematical theory of shock reflection-diffraction, in-

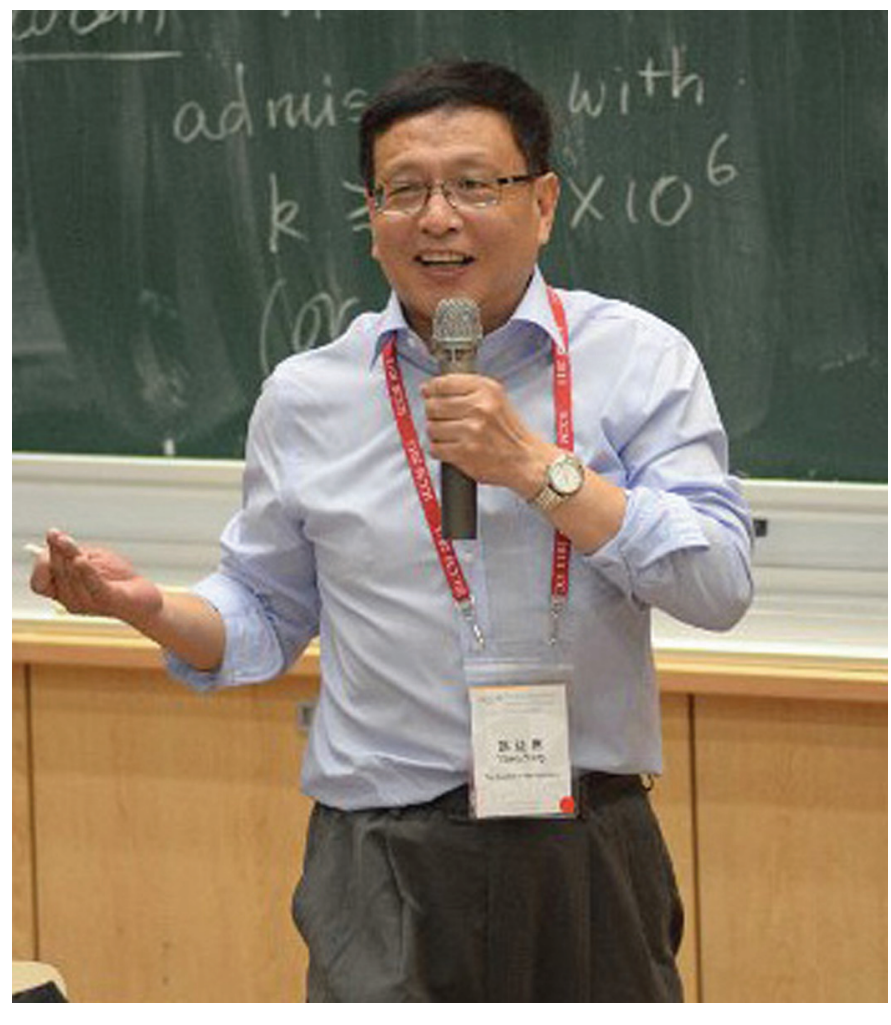

Yitang Zhang

cluding the existence, stability, and regularity of global regular configurations of shock reflection-diffraction by wedges. Further connections, trends, and open problems on the topics will also be addressed.

\section{Bounded gaps between primes}

\section{Yitang Zhang}

The University of New Hampshire

Abstract. Let $p_{n}$ denote the $n$-th prime. It is proved that

$$
\lim _{n \rightarrow \infty} \inf \left(p_{n+1}-p_{n}\right)<7 \times 10^{7} .
$$

The first step of the proof is to reduce the problem to evaluating and comparing certain arithmetic sums, following the recent work of Goldston, Pintz and Yildirim. Then the major problem we encounter is to bound the error terms efficiently. To this end we introduce a stronger version of the Bombieri-Vinogradov theorem 
that is motivated by the work of Bombieri, Friedlander and Iwaniec. Some important results in algebraic geometry are needed to complete the proof.

\section{Dynamics of rational maps}

Guizhen Cui

Academy of Mathematics and Systems Science, Chinese Academy of Sciences

Abstract. In 1980s, Thurston gave a topological characterization for post-critically finite rational maps. It is a fundamental result in complex dynamics with many important applications. In this talk, we will introduce our recent progress about Thurston's theory. We will present the generalization of Thurston theorem to geometrically finite rational maps, and some applications to the structure of Julia sets.

\section{Period integral calculus}

Bong H. Lian

Brandeis University

Abstract. We will discuss an interplay between special function theory and complex geometry. We begin with an example that goes back to Euler, Gauss and Legendre who studied elliptic integrals as solutions to differential equations that bears their names. We will then consider the modern incarnation and generalizations of their constructions-known as period integrals-but from a more geometrical viewpoint. We will describe recent progress in understanding these period integrals and some of their remarkable properties.

\section{Optimal transportation and Monge-Ampere type equation}

Xu-Jia Wang

Australian National University

Abstract. The optimal transportation is to find an optimal mapping of transferring a mass density to another one such that the total cost is minimized. This problem was first introduced by Monge in 1781. Monge's cost function is propositional to the distance the mass is transferred, namely $c(x, y)=|x-y|$, but more general costs are allowed. The optimal transportation has found a variety of applications and has been extensively studied since then. In 1940s Kantorovich introduced a dual functional, and by which, for a large class of cost functions, the optimal mapping can be determined through the potential function satisfying a Monge-Ampere type equation.

The Monge-Ampere equation also arises in many geometric problems and has been studied by Aleksandrov, Calabi, Pogorelov, Cheng-Yau, and Caffarelli, among many others. In this talk we will first introduce the optimal transportation and review the existence of optimal mappings. We then focus on the regularity of the optimal mappings. By studying the Monge-Ampere equation of the potential function, sharp conditions on the cost function have been found by the speaker and his collaborators. For Monge's cost function $|x-y|$, which does not satisfy the sharp conditions, we have also obtained the existence of optimal mappings, and established interesting regularity and singularity results of the mapping.

\section{Local models of Shimura varieties}

Xinwen Zhu

Northwestern University

Abstract. A local model is projective scheme over the ring of integers of a p-adic field which is supposed to étale locally model the integral structure of the Shimura variety of parahoric level structure. Classically, local models were constructed as moduli spaces of linear algebra structures and were studied mostly on a case by case basis. We will report the recent progress of this subject, including the group theoretical definition of local models, their algebro-geometric properties, and the applications.

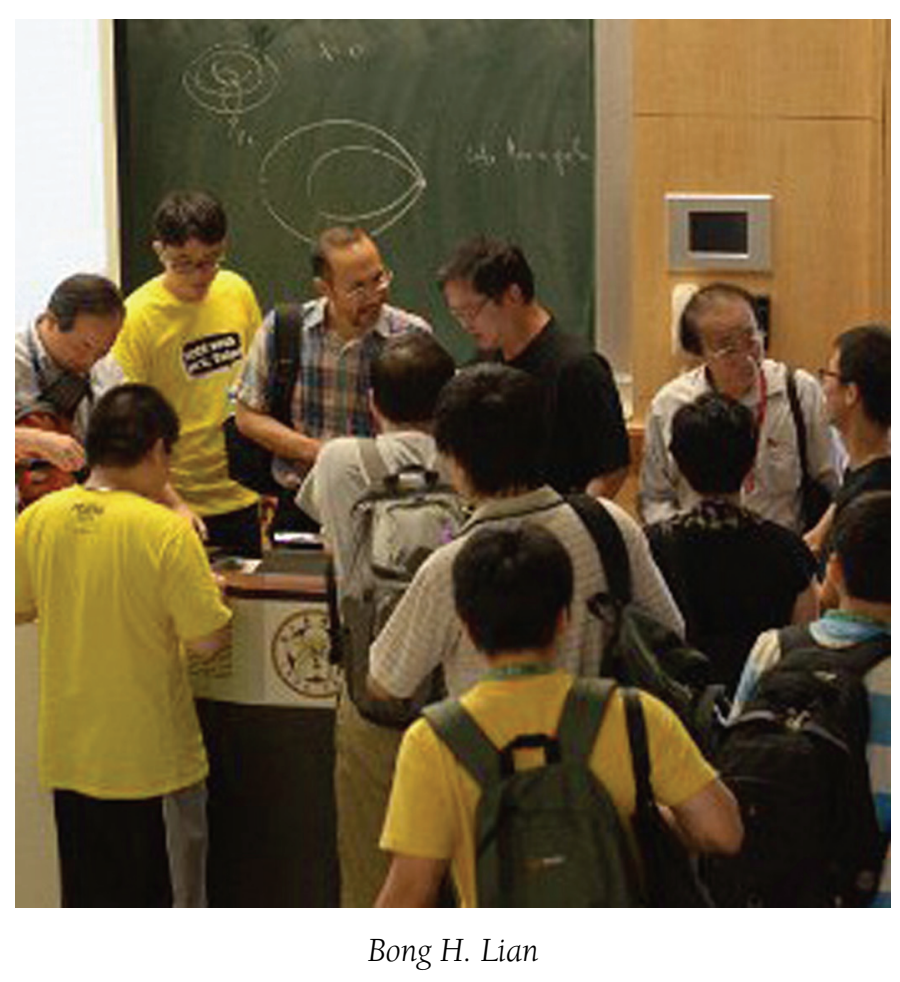

\section{Non-constant CR morphisms between compact strongly pseudo-convex $C R$ manifolds and étale covering between resolutions of isolated singularities}

Stephen Yau

Tsinghua University

Abstract. Strongly pseudoconvex CR manifolds are boundaries of Stein varieties with isolated normal singu- 
larities. We prove that any non-constant CR morphism between two $(2 n-1)$-dimensional strongly pseudoconvex CR manifolds lying in an $n$-dimensional Stein variety with isolated singularities are necessarily a CR biholomorphism. As a corollary, we prove that any non-constant self map of $(2 n-1)$-dimensional strongly pseudoconvex

CR manifold is a CR automorphism. We also prove that a finite étale covering map between two resolutions of isolated normal singularities must be an isomorphism. This is a joint work with Yu-Chao Tu and Huaiqing Zuo.

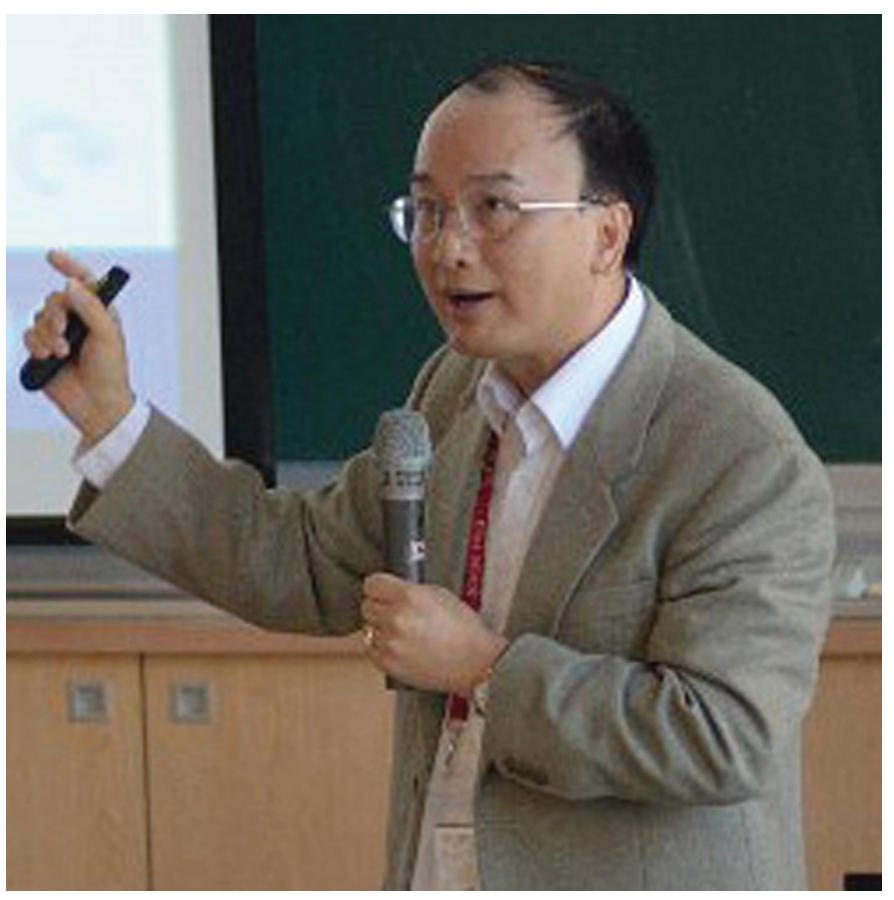

Thomas Yizhao Hou

\section{The interplay between computation and analysis in the study of ClayMillennium Problem on Navier-Stokes equations}

Thomas Yizhao Hou

California Institute of Technology

Abstract. Whether the 3D incompressible Navier-Stokes equations can develop a finite time singularity from smooth initial data with finite energy is one of the Seven Millennium Problems posted by the Clay Mathematical Institute. We review some recent theoretical and computational studies of the 3D Euler equations which show that there is a subtle dynamic depletion of nonlinear vortex stretching due to local geometric regularity of vortex filaments. Our study reveals a surprising nonlinear stabilizing effect that the convection term plays in stabilizing the solution. This is demonstrated through two reduced models of the 3D incompressible Navier-Stokes equations which shows that local flattening of the vortex structure and the effect of convection could lead to dy- namic depletion of the vortex stretching term. Finally we present a new class of solutions to the 3D Euler and Navier-Stokes equations which could lead to a strong nonlinear alignment in the vortex stretching term and have the potential to develop a finite time singularity.

\section{On congruent number problem}

Ye Tian

Morningside Center of Mathematics/Academy of Mathematics and Systems Science, Chinese Academy of Sciences

Abstract. Recall that a positive integer is call a congruent number if it is the area of a right triangle with rational sides. The congruent number problem is to determine if a given positive integer is congruent and if so, find corresponding right triangles. It is known by Heegner that any prime or twice prime congruent to $5,6,7$ modulo 8 is a

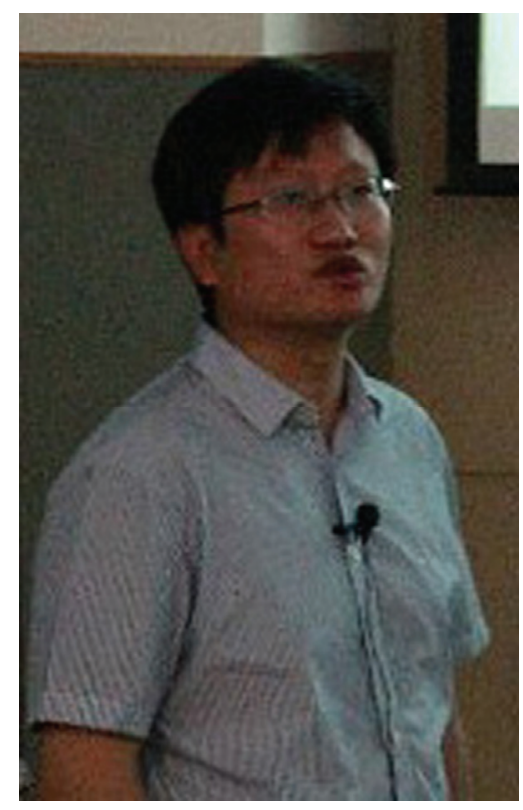

Ye Tian

congruent number. In this lecture, we construct congruent numbers with many prime factors, which extends Heegner's work.

\section{Seiberg-Witten theory and Heegaard Floer theory}

Yi-Jen Lee

The Chinese University of Hong Kong

Abstract. I will outline an equivalence proof of the Seiberg-Witten-Floer homology and the Heegaard Floer homology, and its expected extensions.

\section{Automorphic period, L-function and relative trace formula}

Wei Zhang

Columbia University 
Abstract. We will present some recent progress on the study of period integrals of automorphic forms on classical groups, with an emphasis on their relation to L-functions. There are various approaches; but we will mainly discuss the approach of relative trace formula, started by Jacquet.

\section{Embedded constant mean curvature tori in the threesphere}

Haizhong Li

Tsinghua University

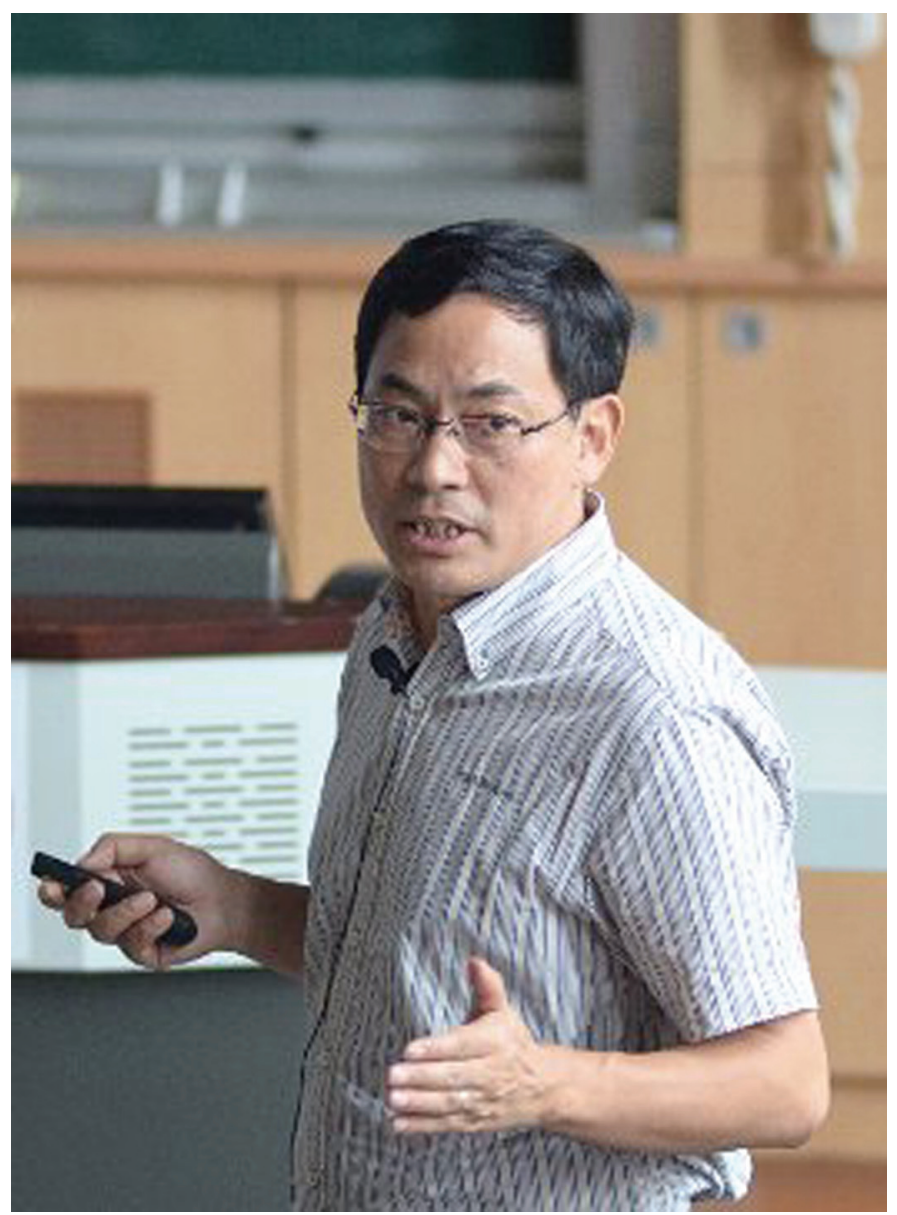

Fuquan Fang

Abstract. The study of constant mean curvature (CMC) surfaces in spaces of constant curvature is one of the classical subjects in differential geometry. There are many beautiful results on this topic. By constructing a holomorphic quadratic differential for CMC surfaces, H. Hopf showed that any CMC two-sphere in Euclidean space is a round sphere. Then S.S. Chern extended Hopf's result to CMC two-spheres in 3-dimensional space forms. The famous Hopf conjecture asks that whether a compact CMC surface in Euclidean space is necessarily a round sphere? In 1980s, H.C. Wente gave a counter example of this conjecture by constructing a compact immersed CMC torus in Euclidean space. In 1950s, A.D. Alexandrov showed that if a compact CMC surface is embedded in Euclidean space, hyperbolic space or a hemisphere, then it must be totally umbilical. The minimal surface is the surface with constant mean curvature zero. It was conjectured by H.B. Lawson in 1970s that the only embedded minimal torus in three-sphere is the Clifford torus. In 1980s, U. Pinkall and I. Sterling conjectured that embedded tori with CMC in three-sphere are surfaces of revolution. In March of 2012, Simon Brendle solved the Lawson conjecture. After that, in April of 2012, Ben Andrews and Haizhong Li gave a complete classification of CMC embedded tori in the three-sphere. When the constant mean curvature is equal to zero or $\pm 1 / \sqrt{3}$, the only embedded torus is the Clifford torus. For other values of the mean curvature, there exists embedded torus which is not the Clifford torus, Ben Andrews and Haizhong Li gave a complete description of such surfaces. As a Corollary, Andrews-Li's Theorem solved the famous Pinkall-Sterling conjecture. In this lecture, we will talk about some basic facts of CMC surfaces and give explanations of key ideas of proofs of Lawson conjecture and Pinkall-Sterling conjecture.

\section{Some mathematical problems in design of experiments}

Ching-Shui Cheng

Institute of Statistical Science, Academia Sinica

Abstract. I will discuss some mathematical problems that arise in statistical design of experiments. Several examples will be used to illustrate connections between design of experiments and finite projective geometry, coding theory and graph theory. Key results will be reviewed and some open questions will be presented.

\section{Geometric transitions and quantum invariance}

Chin-Lung Wang

Taida Institute for Mathematical Sciences

Abstract. A basic question in the study of higher dimensional geometry is to understand the relations of various invariants under geometric transitions among them. For example, the analytic continuation of quantum cohomology under $\mathrm{K}$ equivalent maps is extensively studied in recent years. In dimension 3, the famous Reid fantasy asks if various Calabi-Yau 3-folds are indeed connected via certain extremal transitions. It is even expected that conifold transitions may form the building blocks. This talk surveys some of the developments on the above aspects with emphasize on the relation of quantum A + B models under transitions.

\section{Dispersion and smoothness for two-fluid models in plasma}

Yan Guo

Brown University 
Abstract. Two-fluid models are fundamental fluid models for describing plasma dynamics, in which ions and electrons interact with their self-consistent electromagnetic field. Such an interaction enhances dispersion and leads to global smooth dynamics with no shock formation.

\section{Reflection groups, non-negative curvature and Tits geometry}

Fuquan Fang

Capital Normal University

Abstract. A reflection in a euclidean space (sphere) is one of the fundamental notions of symmetry of geometric figures. It plays a central role in Killing and Cartan's work on Lie algebra in 19th century. Reflections groups on a hyperbolic space is important in hyperbolic geometry, and the first example goes back to F. Klein and Poincare. In this talk I will present

(a) A complete classification of reflection groups and the equivariant structures of complete non negatively curved manifolds; and

(b) A complete classification of positively curved polar manifolds of cohomogeneity at least 2 , which is achieved partially based on Tits geometry.

This is a joint work with Karsten Grove and G. Thorbergsson.

\section{High order numerical methods for convection dominated problems}

Chi-Wang Shu

Brown University

Abstract. Convection dominated partial differential equations are used extensively in applications including fluid dynamics, astrophysics, electro-magnetism, semi-conductor devices, and biological sciences. High order accurate numerical methods are efficient for solving such partial differential equations, however they are difficult to design because solutions may contain discontinuities and other singularities or sharp gradient regions. In this talk we will survey several types of high order numerical methods for such problems, including weighted essentially nonoscillatory (WENO) finite difference methods, WENO finite volume methods, discontinuous Galerkin finite element methods, and spectral methods. We will discuss essential ingredients, properties and relative advantages of each method, and comparisons among these methods. Recent development and applications of these methods will also be discussed.

\section{Enumerative geometry: from classical to modern}

Yu-Jong Tzeng

Harvard University
Abstract. The subject of enumerative geometry goes back at least to the middle of the 19th century. It studies the number of geometric objects in a given class that satisfy a number of incidence or tangency conditions. For example:

(a) How many lines pass through distinct 2 points?

(b) How many lines pass through 4 general lines in 3-space?

(c) How many degree d rational curves on a general quintic 3-fold? (finite?)

The field has been very active in the last twenty years due to its interaction with physics, which provides motivation for many theories and conjectural formulas. One of

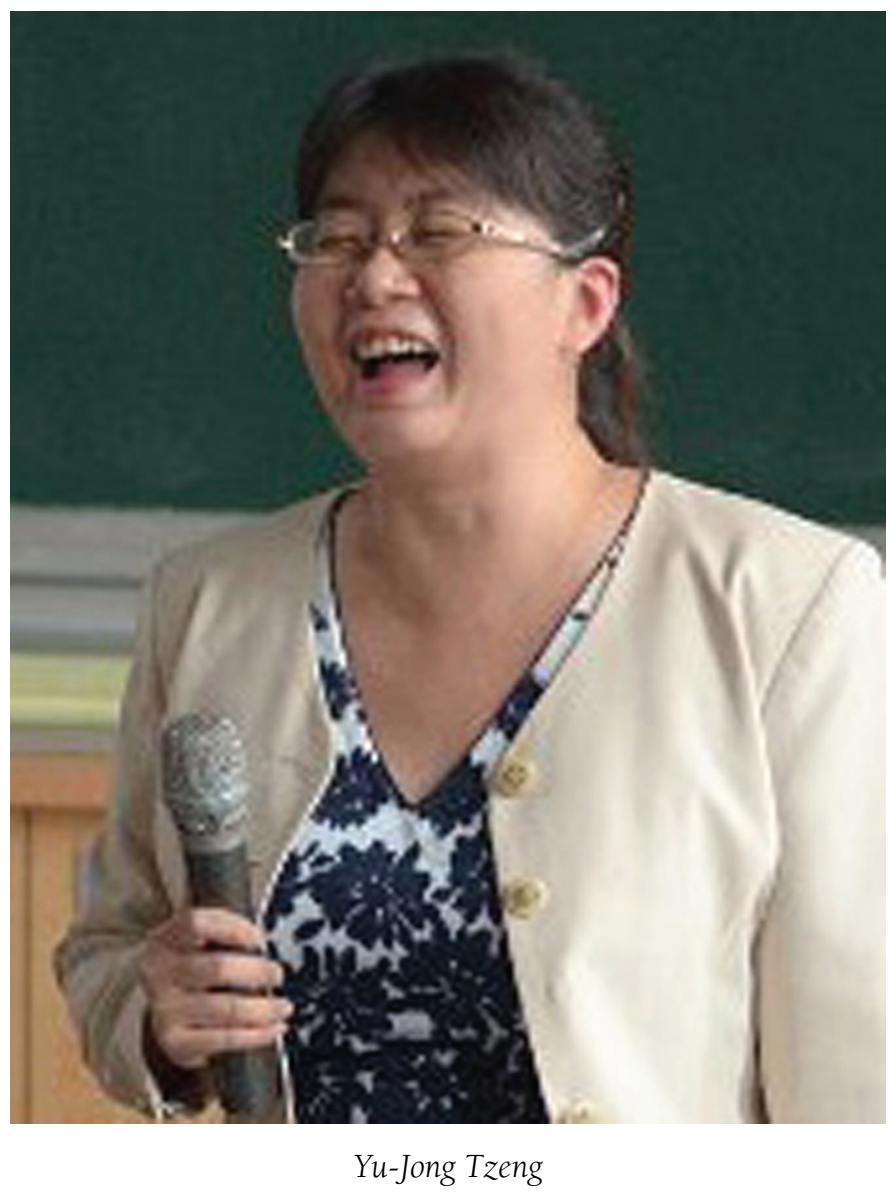

the most famous problem in enumerative geometry is computing Severi degrees, which are the numbers of degree $d$ plane curves with a given number of nodes and pass through an appropriate number of points in general position. The computation of Severi degrees, as well as counting nodal curves on other surfaces are great examples of classical problems which were open for long time and can finally be solved by modern techniques now. Furthermore, a recent conjecture of Gottsche states that there should be a universal formula that computes the number of nodal curves in a sufficiently ample linear system on any surface. In this talk I will first give an indication of what enumerative geometry is about, then give 
a survey about different curve-counting theories. Finally I will focus on the Gottsche's conjecture and its generalizations.

\section{Rigidity in the Langlands correspondence and applications}

Zhiwei Yun

Stanford University

Abstract. In the theory of Langlands correspondence for function fields, rigid automorphic representations and rigid local systems provide explicit and workable examples. We give two applications of such rigid objects. One is the construction of motives over number fields with exceptional Lie groups as Galois groups, answering a question of Serre. The other is the solution of the inverse Galois problem for certain finite groups of Lie type.

\section{Fast-moving finite and infinite trains of solitons and kinks for nonlinear Schrödinger equations}

Tai-Peng Tsai

University of British Columbia

Abstract. I will first review known results on multi-solitons of dispersive partial differential equations, which are special solutions behaving like the sum of many weakly-interacting solitary waves. I will then describe my recent joint work with Dong Li and Stefan Le Coz: Assuming the composing solitons have sufficiently large relative speeds, we prove the existence and uniqueness of a soliton train which is a multi-soliton composed of infinitely many solitons. We also give a new construction of multi-solitons and prove uniqueness in an exponentially small neighborhood. In the 1D case, we can add to the infinite train an additional half-kink, which is a solution with a non-zero background at minus infinity.

\section{Hall algebras and quantum groups arising from period 2 derived categories}

Jie Xiao

Tsinghua University

Abstract. Inspired by the recent work of Bridgeland [1], we extend the derived Hall algebras defined in [2] and [3] to that from a period 2 derived category. This gives a successful model to realize the quantum groups globally. The talk is based on a joint work with X. Chen and F. Xu.

\section{Universality of random matrices, Dyson's Brownian and De Giorgi-Nash-Moser theory of parabolic regularity}

Horng-Tzer Yau

Harvard University

Abstract. Eugene Wigner's revolutionary vision predicted that the energy levels of large complex quantum systems exhibit a universal behavior: the statistics of energy gaps depend only on the basic symmetry type of the model. These universal statistics show strong correlations in the form of level repulsion and they represent a new paradigm of point processes that are characteristically different from the Poisson statistics of independent points.

Simplified models of Wigner's thesis have recently become mathematically accessible. For mean field models represented by large random matrices with independent entries, the celebrated Wigner-Dyson-Gaudin-Mehta (WDGM) conjecture asserts that the local eigenvalue statistics are universal. For invariant matrix models, the eigenvalue distributions are given by a log-gas with potential $V$ and inverse temperature $\beta=1,2,4$. For $\beta \notin\{1,2,4\}$, there is no natural random matrix ensemble behind this

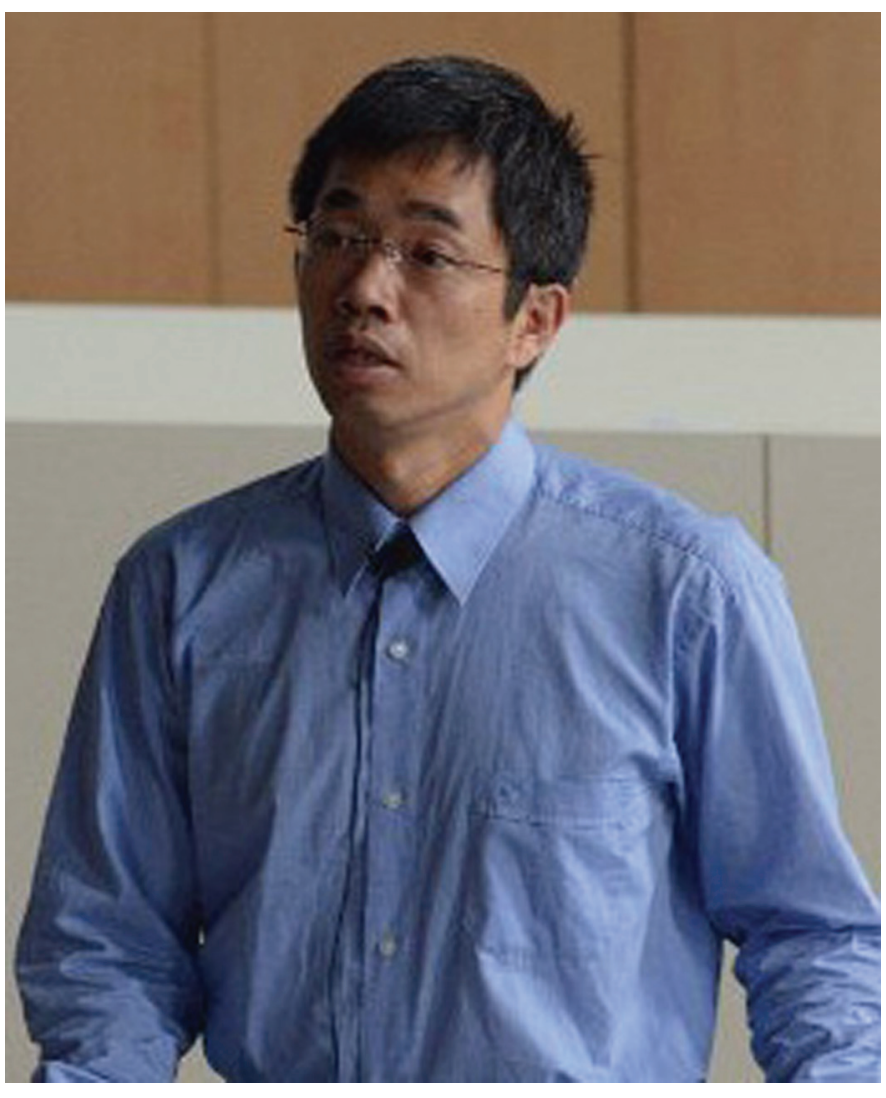

Tai-Peng Tsai

model, but the analogue of the WDGM conjecture asserts that the local statistics are independent of $V$.

In this lecture, we review the recent solution to these conjectures for both invariant and non-invariant ensembles.

We will demonstrate that the local ergodicity of the Dyson Brownian motion is the intrinsic mechanism behind the universality. Furthermore, we will show that the universality of gap distribution requires to prove a Holder regularity of a discrete parabolic equation with random coefficients. For this purpose, we incorporate the ideas of parabolic regularity via a De Giorgi-Nash-Moser approach. 


\section{Representation theories of Lie algebras and Lie superalgebras}

Shun-Jen Cheng

Institute of Mathematics, Academia Sinica

Abstract. We shall discuss the representation theories of Lie algebras and Lie superalgebras of classical types, and explain how these two apparently different theories are connected.

\section{Structure-preserving algorithms for Riccati-type} equations: rediscovery, redevelopment and applications

Wen-Wei Lin

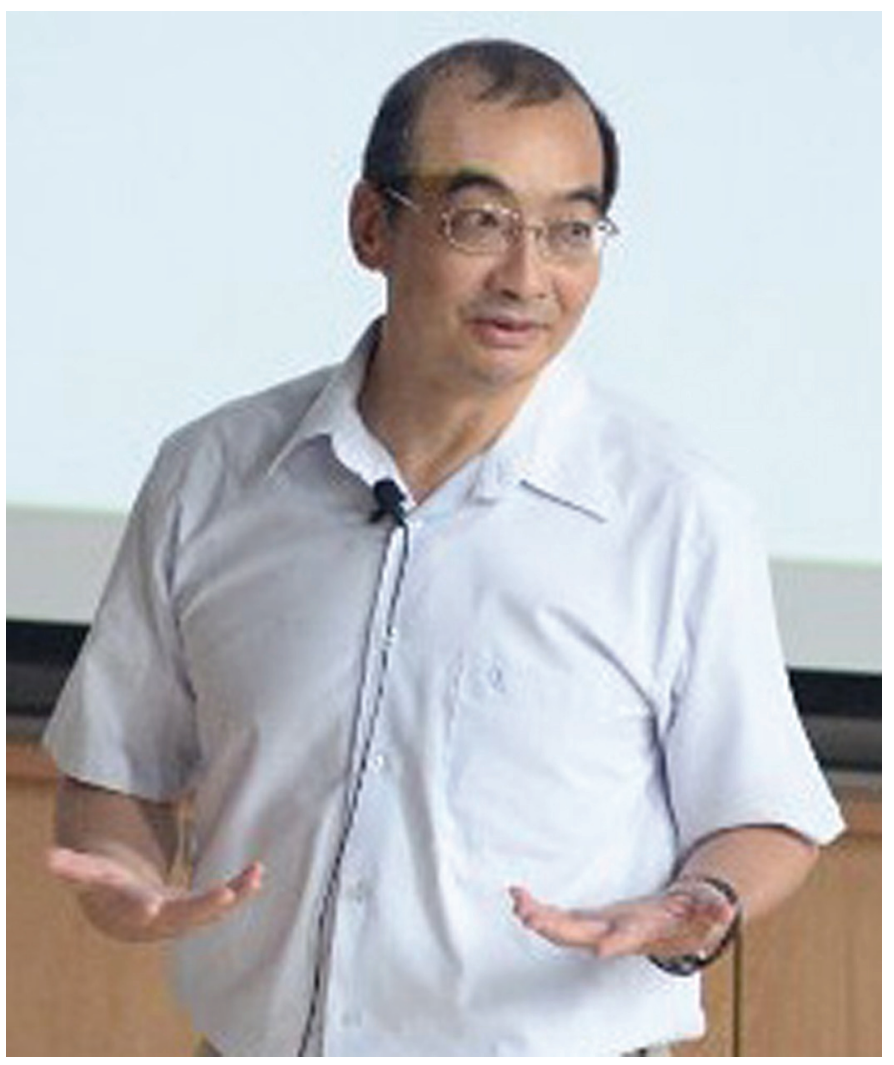

Horng-Tzer Yau

\section{National Chiao Tung University}

Abstract. In this talk, we attempt to tell the interesting story about the recent rediscovery and revival of the once faded and almost forgotten doubling algorithm for the discrete-time algebraic Riccati equation. Armed with some new insight and theoretical redevelopment, the method, now called the structure-preserving doubling algorithm (SDA), is first linked to palindromic eigenvalue problems. Generalizations were then developed for continuous-time, nonsymmetric and PCP algebraic Riccati equations and nonlinear matrix equations, for a vast array of applications in vibration analysis, surface acoustic wave simulation, optimal control, stochastic systems, transport theory, time-delayed systems, nano research, etc. We shall present some key theoretical results associated with selected important applications, and a summary of the recent discovery that the SDA can be adapted to solve large-scale problems of dimension $n$, with an $O(n)$ computational complexity and memory requirement. 